\title{
Tiaogan Qingxin Granule treatment increases myocardial connexin 43 expression in a rat model of arrhythmia
}

\author{
J.H. Yao, C.H. Qu, L. Ma and X.Z. Chang \\ Department of Traditional Chinese Medicine, Yantaishan Hospital, Yantai, \\ China \\ Corresponding author: X.Z. Chang \\ E-mail: yaojh8855@126.com \\ Genet. Mol. Res. 15 (2): gmr.15027934 \\ Received October 28, 2015 \\ Accepted February 12, 2016 \\ Published June 3, 2016 \\ DOI http://dx.doi.org/10.4238/gmr.15027934
}

\begin{abstract}
Myocardial ischemia-induced arrhythmia, especially ventricular arrhythmia, is the main reason for sudden cardiac death. Therefore, ischemic ventricular arrhythmia-targeted treatments are urgently needed. The mechanism of Tiaogan Qingxin Granule in premature ventricular beat (PVB) treatment was explored in arrhythmic rats pretreated with Tiaogan Qingxin Granule. Sprague-Dawley rats $(\mathrm{N}=40)$ were randomly divided into 4 groups: sham-operated, arrhythmia model, Wenxin Granule, and Tiaogan Qingxin Granule. The ischemic arrhythmia model was established by ligating the left anterior descending coronary artery. The Tiaogan Qingxin Granule group was treated intragastrically for 7 days before surgery. Sham-operated rats underwent thoracotomy without coronary artery ligation. Myocardial infarction rate was measured using the triphenyltetrazolium chloride method and $\mathrm{Cx} 43$ expression was quantified by western blotting. Compared to the arrhythmia model group, the Tiaogan Qingxin Granule group showed a significant reduction in the myocardial infarct size and myocardial infarction rate $(\mathrm{P}<0.01)$. Cx43 expression in the left ventricular myocardial tissues was significantly lower in the
\end{abstract}


arrhythmia model group than in the sham-operated group $(\mathrm{P}<0.01)$, but significantly higher in the Tiaogan Qingxin Granule group $(\mathrm{P}<$ 0.01). Intergroup difference in the relative $\mathrm{Cx} 43$ expression between the Tiaogan Qingxin Granule and Wenxin Granule groups was not significant $(\mathrm{P}>0.05)$. Thus, Tiaogan Qingxin Granule reduced the myocardial infarct size, lowered the myocardial infarction rate, and increased $\mathrm{Cx} 43$ expression, possibly by increasing blood supply to the cardiac muscles. In conclusion, Tiaogan Qingxin Granule may be useful for treating ischemic PVB.

Key words: Tiaogan Qingxin Granule; Myocardial infarction; Ischemic premature ventricular beats; Animal models

\section{INTRODUCTION}

Millions of people worldwide succumb to SCD, which accounts for $15-20 \%$ of all deaths. Similarly, it is a leading cause of death in China (Li, 2009). A 2009 study conducted at the Beijing Fuwai Hospital showed that more than 544,000 Chinese die from SCD every year, and the incidence is increasing (Hua et al., 2009). Most SCDs are caused by ventricular arrhythmia (Matsushita al., 2006), particularly by ischemic ventricular arrhythmia. Therefore, further elucidation of the pathogenesis as well as the development of targeted treatment strategies for this disease are urgently needed.

Abnormal expression and distribution of myocardial gap junction connexins, which are the main constituent proteins of ventricular gap junctions, leads to abnormalities in ventricular myocytes. For example, changes in conduction velocity and conduction anisotropy can result in reentry and conduction block arrhythmias (Chen et al., 2015). Among the connexins, the abnormalities of $\mathrm{Cx} 43$ are closely related to the occurrence and persistence of a variety of arrhythmias (Wang et al., 2013a). Therefore, the correlation of dephosphorylation and degradation of myocardial $\mathrm{Cx} 43$ during myocardial ischemia-reperfusion (MIR) with the incidence of arrhythmia has been extensively investigated by clinical researchers (Kostin et al., 2004; Li et al., 2010).

PVB is one of the most common types of clinical arrhythmias. In recent years, a number of compounds, anti-arrhythmic herbs, and herbal extracts have been investigated for their anti-arrhythmic effects (Xin et al., 2011). For example, the Jianxin Pinglv Pill enhanced $\mathrm{Cx} 43$ expression in the subepicardial ischemic myocardium, thereby antagonizing ischemiareperfusion injury (He et al., 2004). The Fuzhenghuayu Capsule showed partial improvement in $\mathrm{Cx} 43$ remodeling of the myocardium in rats with myocardial infarction. It also improved ventricular fibrillation threshold (VFT) in a ventricular remodeling system (Wu et al., 2007). The compounds Shuangshen Tongguan Recipe and Fumai Decoction inhibited the degradation of $\mathrm{Cx} 43$ and improved the communication between myocardial cells (Liu et al., 2005; Gong and Cheng, 2014). Similarly, the Gegen Qinlian Decoction showed an increase in the expression of $\mathrm{Cx} 43$ in cardiac muscles and improved the remodeling of pathological changes in myocardial tissue to achieve an anti-arrhythmic effect (Yang, 2013). Most of the previously reported compound recipes were composed of Yiqi Huoxue pharmaceutical agents. However, the antiPVB mechanism of the compound preparations of traditional Chinese medicines composed of Huatan Huoxue pharmaceutical agents has not been reported.

The Buchangwenxin Granule is the first state-approved anti-arrhythmic Chinese 
patent drug. It was developed by the China Academy of the Chinese Medical Sciences and is produced by the Buchang Pharmaceutical Company. Its indications include atrial fibrillation, premature beats, and sinus tachycardia resulting from the deficiency of qi and yin. The main ingredients of the Buchangwenxin Granule are dangshen (Radix Codonopsis), sanqi (Radix Notoginseng), solomonseal rhizome (Rhizoma Polygonati), amber, and nardostachys root (Radix et Rhizoma Nardostachyos) (Li et al., 2011). Pharmacological studies suggest that dangshen and solomonseal rhizome strengthen the spleen, benefit qi, and nourish yin, which can significantly increase myocardial contractility. Sanqi and nardostachys root strengthen the spleen, regulate qi-flow, remove blood stasis, dilate blood vessels, increase coronary blood flow, and reduce myocardial oxygen consumption and blood viscosity. In addition, amber promotes water and blood circulation, leading to diuresis, calming, and sedative effects (Wang et al., 2011). Furthermore, a recent study has shown that Buchangwenxin Granule mediated anti ischemic arrhythmia mechanism through enhanced expression of $\mathrm{Cx} 43$ (Guo et al., 2014).

Tiaogan Qingxin Granule is a proprietary Chinese medicine recently developed by the manufacturing laboratory at the Yantaishan Hospital. The constituents of Tiaogan Qingxin Granule include Chinese thorowax root (Radix Bupleuri), orange fruit (Fructus Aurantii), turmeric root tuber (Radix curcumae), red peony root, golden thread (Rhizoma Coptidis), pinellia tuber (Rhizoma Pinelliae), whole snakegourd fruit, lightyellow sophora root (Radix Sophorae Flavescentis), kudzuvine root (Radix Puerariae), grassleaf sweetflag rhizome (Rhizoma Acori Tatarinowii), nacre (Concha Margaritifera), stir-baked jujube kernel, and prepared licorice root. The indications for which Tiaogan Qingxin Granule is used include sinus tachycardia, premature atrial and ventricular beats, and other symptoms caused by stagnation of liver-qi, phlegm and blood stasis (Yao and Gao, 2007). Modern pharmacological studies show that saikosaponins, the main effective ingredient of Chinese thorowax root, have a strong sedative effect; orange fruit significantly reduces coronary resistance, increases coronary blood flow, and reduces myocardial oxygen consumption; snakegourd fruit prolongs the action potential and exerts an anti-arrhythmic effect; peony root water extract increases myocardial nutrition and blood flow; and berberine from golden threads significantly enhances atrial contraction and slows the sinus rate. It also prevents and assists in treating coronary artery ligation-induced ventricular arrhythmias in animals. Additionally, berberine reduces cardiac automaticity, prolongs the action potential duration and effective refractory period, eliminates the reentry impulse, inhibits myocardial fast $\mathrm{Na}+$ influx, and possibly blocks $\mathrm{Ca} 2+$ channels, all of which lead to anti-arrhythmic effect. Lastly, pinellia tuber demonstrated an anti-arrhythmic effect (Chen et al., 2002), but its mechanism of action has not been reported.

In this study, myocardial ischemia was induced by left anterior descending coronary artery ligation in rats. The effect of Tiaogan Qingxin Granule on myocardial Cx43 expression was investigated after pretreatment with test compounds administered intragastrically prior to induction of ischemic arrhythmia. The anti-PVB mechanism of Tiaogan Qingxin Granule in terms of reducing phlegm and promoting blood circulation was also investigated.

\section{MATERIAL AND METHODS}

\section{Animals}

Forty specific pathogen-free (SPF) male Sprague Dawley (SD) rats weighing $200 \pm$ $20 \mathrm{~g}$ were obtained from the SPF animal laboratory of Binzhou Medical College [Certificate 
of Quality No.: SCXK (Beijing) 2011-0009]. An approval was obtained from the Institute of Animal Ethics of Yantaishan Hospital for the surgical procedures.

\section{Grouping and modeling}

SD rats (40) were randomly divided into four groups: the sham-operated, arrhythmia control, Wenxin Granule, and Tiaogan Qingxin Granule groups. The surgical methods and dosages were selected as reported in literature (Kuniyasu et al., 2003; Yang, 2013). The shamoperated and arrhythmia control groups received an isovolemic $0.9 \%$ sodium chloride solution intragastrically. The Wenxin Granule (Cat no.: Z10950026) group received $1 \mathrm{~mL}$ Granule/100 $\mathrm{g}$ body weight (equivalent to $4.28 \mathrm{~g}$ crude drug/kg body weight) intragastrically, once per day, beginning 7 days before surgery. The Tiaogan Qingxin Granule group was given $1 \mathrm{~mL}$ Granule/100 g body weight (equivalent to $4.32 \mathrm{~g}$ crude drug/kg body weight) intragastrically, once per day, beginning 7 days before surgery. Two weeks after treatment, the rats were weighed and anesthetized by intraperitoneal injection of $40 \mathrm{mg} / \mathrm{kg}$ pentobarbital sodium. The rats were immobilized in the supine position on a pre-warmed stage and tracheotomies were performed. The rat limbs were subcutaneously connected to a BL-420E biological and functional experimental system and standard lead II electrocardiography (ECG) was performed. A thoracotomy was performed at the level of third-fourth intercostal space at the left side of the sternum and the heart was extruded through the incision. A silk suture (No. 1) was threaded underneath the anterior descending coronary artery, and the heart was placed back. The suture was alternatively tautened and released, for $5 \mathrm{~min}$ each, and this procedure was repeated three times. ST-segment elevation in ECG monitoring indicated successful ischemia. The sham-operated group also underwent thoracotomy, but coronary artery ligation was not performed. After the third ischemic treatment, reperfusion was performed for $4 \mathrm{~h}$. Then, the rats were euthanized and the heart was removed. The left ventricle was excised and cut in two parts. One part was fixed in 10\% neutral buffered formalin and the other stored in liquid nitrogen for subsequent analysis.

\section{Observed indicators}

\section{Determination of the rate of myocardial infarction}

Rats were sacrificed immediately after surgery. The hearts were excised by thoracotomy, washed with ice-cold saline, dried with filter paper, and processed for TTC staining. The atria, right ventricle, and connective tissues were removed to expose the left ventricle. The left ventricle was cut from the apex to the base into uniform slices (approximately $2 \mathrm{~mm}$ thick) with a scalpel. These slices were immediately placed in a water bath, at $37^{\circ} \mathrm{C}$, containing $1 \%$ TTC solution (Boster, Wuhan, China) (in PBS, pH 7.4) for staining to reveal areas of infarction. The samples were then removed from the staining solution, and the infarcted areas were snipped out. The retained water was blotted, and the slices were weighed. The rate of myocardial infarction was calculated by dividing the myocardial infarct weight (MIW, g) by the total heart weight (HW, g) x 100\%.

\section{Cx43 expression}

The expression of $\mathrm{Cx} 43$ in the left ventricular myocardium was measured by western blotting. Myocardial tissue specimens were homogenized, the supernatants were collected, 
and the protein concentration was determined by the Lowry method. The proteins were diluted with $0.9 \% \mathrm{NaCl}$ to obtain same concentration and same amounts were denatured at $100^{\circ} \mathrm{C}$ for $3 \mathrm{~min}$. Proteins were then separated by $10 \%$ sodium dodecyl sulfate - polyacrylamide gel electrophoresis (SDS-PAGE), transferred to PVDF membranes, stored at $4^{\circ} \mathrm{C}$ for $80 \mathrm{~min}$, and blocked with 5\% TBST in skim milk for $1 \mathrm{~h}$. Subsequently, the membranes were treated with rabbit anti-mouse Cx43 polyclonal primary antibody (diluted 1:1,000 in TBST; Cell Signaling Technology, Danvers, MA, US) at $4^{\circ} \mathrm{C}$ overnight, washed, and incubated with horseradish peroxidase-conjugated secondary antibody (diluted 1:2,000; ZSGB-BIO, Beijing, China) for $1 \mathrm{~h}$ at room temperature. Thereafter, the membranes were thoroughly washed, and treated with enhanced chemiluminescence (ECL) reagent to develop the signal. The results were recorded on X-ray films and densitometric analysis was performed using Image $\mathrm{J}$ software to determine the density of the hybridized bands. GAPDH $(1: 1,000)$ was used as an internal reference for the calculation of the expression level of $\mathrm{Cx} 43$.

\section{Statistical analysis}

Data are reported as means \pm standard deviation and analyzed with SPSS 16.0. Differences between two groups were compared by the $t$-test, and $\mathrm{P}<0.05$ was considered statistically significant.

\section{RESULTS}

\section{Effect of Tiaogan Qingxin Granule on the infarct size and myocardial infarction rate in arrhythmia model rats}

After ischemia-reperfusion, rats were sacrificed and dissected to obtain the hearts, which were stained with TTC. The myocardium of the sham-operated group stained rosy red, indicating no infarct zone. However, parts of the myocardium in the arrhythmia model group showed a pale color, indicating a large infarct area and severe myocardial infarction as shown in Figure 1. A significant difference $(\mathrm{P}<0.01)$ in the infarct size and infarction rate of the sham group as compared to the arrhythmia model group indicated proper establishment of the arrhythmia model. Additionally, the Wenxin, and Tiaogan Qingxin Granule groups showed significant infarct size reduction $(\mathrm{P}<0.01)$ as compared to the arrhythmia model group, with no significant difference between the two treated groups $(\mathrm{P}>0.05)$ as shown in Table 1 .

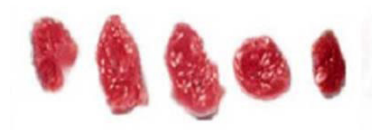

A

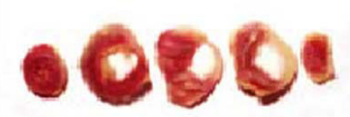

$\mathrm{C}$

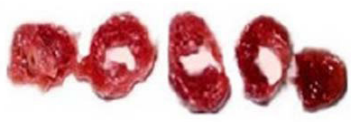

$\mathrm{B}$

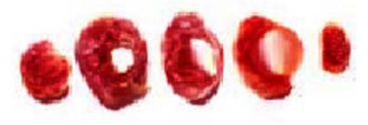

$\mathrm{D}$

Figure 1. TTC staining for measurement of myocardial infarction rates. A. Sham-operated group. B. Arrhythmia model group. C. Wenxin Granule group. D. Tiaogan Qingxin Granule group. 
Table 1. Effect of Tiaogan Qingxin Granule on myocardial infarct size and infarction rate in arrhythmia model rats (mean \pm s.d., $\mathrm{N}=10$ ).

\begin{tabular}{l|c|c|c}
\hline Group & Infarct size $\left(\mathrm{mm}^{2}\right)$ & Infarct area/heart (\%) & Myocardial infarction weight $(\mathrm{g})$ \\
\hline Sham & 0 & 0 & 0 \\
\hline Model & $88.36 \pm 18.27$ & $23.58 \pm 4.43$ & $0.208 \pm 0.046$ \\
\hline Wenxin & $70.02 \pm 16.96^{*}$ & $18.50 \pm 2.84^{*}$ & $0.162 \pm 0.045^{*}$ \\
\hline TiaoganQingxin & $62.95 \pm 19.93^{* *}$ & $19.06 \pm 3.36^{*}$ & $0.157 \pm 0.033^{*}$ \\
\hline
\end{tabular}

Compared to the model group, $* \mathrm{P}<0.05, * * \mathrm{P}<0.01$.

\section{Effect of Tiaogan Qingxin Granule on myocardial Cx43 expression in arrhythmia model rats}

The density of the $\mathrm{Cx} 43$ bands in each group is shown in Figure 2, and the relative expression is provided in Table 2. Myocardial Cx43 expression in the ischemic arrhythmia model group was significantly lower than in the sham-operated group $(\mathrm{P}<0.01)$. After treatment, $\mathrm{Cx} 43$ expression in the Wenxin Granule and Tiaogan Qingxin Granule groups was significantly increased as compared to that observed in the model group $(\mathrm{P}<0.01)$. No significant difference was found between the Wenxin Granule group and the Tiaogan Qingxin Granule group $(\mathrm{P}>0.05)$.

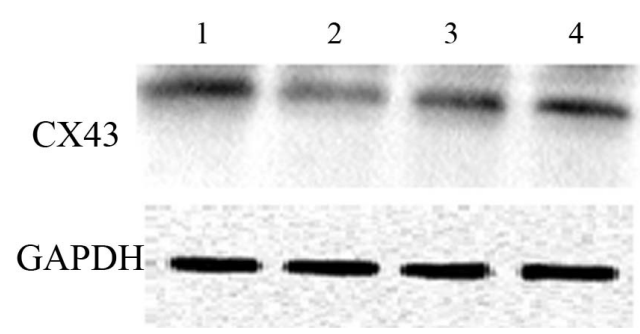

Figure 2. Western blots of $\mathrm{Cx} 43$ and GAPDH proteins in each group. Lane 1, Sham-operated group. Lane 2, Arrhythmia model group. Lane 3, Wenxin Granule group. Lane 4, Tiaogan Qingxin Granule group.

Table 2. Effect of Tiaogan Qingxin Granule on myocardial Cx43 expression in arrhythmia model rats $(\mathrm{N}=10)$.

\begin{tabular}{l|c|c|c}
\hline Group & GAPDH & Density of the bands (mean $\pm \mathrm{SD}, \mathrm{N}=10)$ & Relative content of Cx43 (Cx43/GAPDH) \\
\hline Sham & 148.25 & $247.49 \pm 3.53$ & $1.67 \pm 0.02$ \\
\hline Model & 152.10 & $141.79 \pm 19.56^{\# \#}$ & $0.93 \pm 0.13^{\# \#}$ \\
\hline Wenxin & 143.75 & $165.72 \pm 10.40^{* *}$ & $1.15 \pm 0.07^{* *}$ \\
\hline TiaoganQinxin & 149.05 & $176.45 \pm 12.50^{* *}$ & $1.18 \pm 0.08^{* *}$ \\
\hline
\end{tabular}

Compared with the sham-operated group, ${ }^{\#} \mathrm{P}<0.05,{ }^{\#} \mathrm{P}<0.01$; compared with the arrhythmia model group, ${ }^{*} \mathrm{P}<$ $0.05, * * \mathrm{P}<0.01 . \mathrm{SD}=$ standard deviation.

\section{DISCUSSION}

Arrhythmia is one of the most serious conditions among the cardiovascular diseases, and also one of the main causes of SCD. In this study, left anterior descending coronary artery ligation was performed to induce ischemia-reperfusion injury and arrhythmia to investigate the mechanism of Granule action in rats. The effect of Tiaogan Qingxin Granule on the myocardial gap junction protein $\mathrm{Cx} 43$ expression was studied after intragastric administration of the granules as pretreatment. 
The incidence of arrhythmia in patients with ischemic heart disease is positively correlated to the size of low-voltage areas; the larger the area of myocardial infarction, the greater the size of the myocardial low-voltage area. Therefore, the size of the infarct directly affects the incidence of ventricular arrhythmias (Tosaki et al., 1996). Currently, the most common method to determine the infarct size is TTC staining. It is a classic and reliable method, which detects the dehydrogenase activity within tissues. The activity of dehydrogenase within the necrotic myocardial tissues of rats is reduced by myocardial infarction caused by left anterior descending coronary artery ligation. Therefore, the infarcted myocardial tissue is not stained, whereas the normal myocardium is stained brick red. This apparent color contrast makes the myocardial infarction area easy to identify, and measurement of the myocardial infarction rate is more objective and accurate (Garcia-Dorado et al., 2002). In this study, the infarct size of the ischemic myocardium was determined by the TTC staining method, and the myocardial infarction rate was obtained by calculating the ratio of the weight of the ischemic myocardium to the total weight of the heart. This approach allowed to investigate the effect of Tiaogan Qingxin Granule on the extent of myocardial ischemia from a macroscopic perspective. The results showed that Tiaogan Qingxin Granule significantly reduced the myocardial infarct size and infarction rate as compared to the arrhythmia model group $(\mathrm{P}<0.01)$. However, there was no significant difference in the infarct size and infarction rate as compared to the Wenxin Granule group $(\mathrm{P}>0.05)$. Wang and Liu explored the effect of Wenxin Granule on cardiomyocytes during MIR injury and found that it significantly reduced the infarct size in a rat model (Wang and Liu, 2014). Results of the present study are consistent with the findings of Wang and Liu. To date, there are no reports on the effect of Tiaogan Qingxin Granule on myocardial infarction in myocardial ischemic model rats. Results of this study suggest that Tiaogan Qingxin Granule provides protection against ischemic myocardial injury.

Cx43 anomalies are closely related to the occurrence and persistence of various arrhythmias (Wang et al., 2013b). MIR causes intracellular calcium influx and an increase in the intracellular calcium concentration that results in activation of proteases, and induces the release of lysosomes from cells (Fong et al., 2013). Concurrently, calcium acts as a second messenger in intercellular signal transduction and induces the activation of calcineurin. It leads to the dephosphorylation of the 368 locus in the $\mathrm{C}$-terminus of the $\mathrm{Cx} 43$ protein and promotes the degradation of $\mathrm{Cx} 43$ in myocardial tissues. As a result, the $\mathrm{Cx} 43$ concentration in the myocardium is decreased and its distribution is disturbed, which interrupts the electrical conductance of myocardial tissues, resulting in loss of coupling between cardiomyocytes (Waza et al., 2014). In such instances, the overall conduction velocity of the cardiac tissue is decreased and a unidirectional block is induced. Changes in the direction of cardiac conduction between cells lead to conduction block and reentry, and eventually, arrhythmias (Wang et al., 2013b). In addition, cardiomyocyte apoptosis may also result in reduced expression of $\mathrm{Cx} 43$ in myocardial tissues with MIR. The decreased expression and disturbed $\mathrm{Cx} 43$ distribution across myocardial cells after MIR constitute the initial stage of cardiac reconstruction at the molecular level, which is also the basis for arrhythmia (Peters et al., 1997). In this study, western blotting was used to investigate the effect of Tiaogan Qingxin Granule on left ventricular myocardial $\mathrm{Cx} 43$ expression. The densitometry method was used to quantify the bands. Results showed that after the intervention with Tiaogan Qingxin Granule, the degree of $\mathrm{Cx} 43$ degradation was reduced in the ischemic area. The outcomes of the Wenxin Granule group and the Tiaogan Qingxin Granule group were similar, with no significant difference in the relative content of $\mathrm{Cx} 43$ protein $(\mathrm{P}>0.05)$; however, significant differences were observed 
as compared to the model group $(\mathrm{P}<0.01)$. These results are consistent with previous reports (Lin et al., 2003; Sun et al., 2005; Yu and Sheng, 2011). Results of this study indicate that the possible mechanism of action of Tiaogan Qingxin Granule is restoration of the abnormal Cx43 expression by improvement in the myocardial blood supply that results in stabilized electrical coupling between cardiomyocytes after MIR. Therefore, it can serve as a potential treatment for ischemic PVB (Beardslee et al., 2000; Dupont et al., 2001).

Because of the limited data for these experimental research conditions, there are some caveats to this study. For instance, the relationship between $\mathrm{Cx} 43$ expression and arrhythmias, the effect of Tiaogan Qingxin Granule treatment on regulatory genes, and how upstream regulatory genes modulate the expression of $\mathrm{Cx} 43$ are yet to be explored.

Current study showed that Tiaogan Qingxin Granule pretreatment could reduce infarct size and the rate of myocardial infarction. It is suggested that these effects of Tiaogan Qingxin Granule on ischemic PVB were caused by the increased expression of $\mathrm{Cx} 43$ protein. Hence, the results of this study provide a molecular basis and proof of evidence-based medicine for the clinical application of Tiaogan Qingxin Granule in the prevention and treatment of ischemic PVB.

\section{Conflicts of interest}

The authors declare no conflict of interest.

\section{ACKNOWLEDGMENTS}

Research supported by the "2015" Scientific and Technological Research Program of Chinese Medicine of Shandong Province (\#2015-421).

\section{REFERENCES}

Beardslee MA, Lerner DL, Tadros PN, Laing JG, et al. (2000). Dephosphorylation and intracellular redistribution of ventricular connexin43 during electrical uncoupling induced by ischemia. Circ. Res. 87: 656-662. http://dx.doi. org/10.1161/01.RES.87.8.656

Chen Q, Liu QY and Chen ChX (2002). Pharmacology of Chinese Traditional Medicine 2nd edn (in Chinese). People's Medical Publishing House, Beijing.

Chen ZY, Wang R, Huang F, Yuan DD, et al. (2015). Inhibition of gap junctions relieves the hepatotoxicity of TNF-a. Genet. Mol. Res. 14: 11896-11904. http://dx.doi.org/10.4238/2015.October.5.3

Dupont E, Matsushita T, Kaba RA, Vozzi C, et al. (2001). Altered connexin expression in human congestive heart failure. J. Mol. Cell. Cardiol. 33: 359-371. http://dx.doi.org/10.1006/jmcc.2000.1308

Fong JT, Kells RM and Falk MM (2013). Two tyrosine-based sorting signals in the Cx43 C-terminus cooperate to mediate gap junction endocytosis. Mol. Biol. Cell 24: 2834-2848. http://dx.doi.org/10.1091/mbc.E13-02-0111

Garcia-Dorado D, Ruiz-Meana M, Padilla F, Rodriguez-Sinovas A, et al. (2002). Gap junction-mediated intercellular communication in ischemic preconditioning. Cardiovasc. Res. 55: 456-465. http://dx.doi.org/10.1016/S0008$\underline{6363(02) 00441-8}$

Gong YP and Cheng Z (2014). Study the effect of Fumai decoction on the incidence of arrhythmia in rat myocardial ischemia model and the expression of Cx43. J. Yunnan Univ. Tradit. Chin. Med. 27: 4-7.

Guo L, Zhao LY and Zhao FJ (2014). Effects of Wenxin Granule on the expression of small RNA-1 and its regulatory protein in ischemic arrhythmic rats. China J. Tradit. Chin. Med. Pharm. 7: 2346-2349.

He XP, Chen DF, Zheng JH and Du SH (2004). Mechanism of Jianxin Pinglv Pill in counteracting arrhythmia. $J$. Guangzhou Univ. Tradit. Chin. Med. 12: 281-283.

Hua W, Zhang LF, Wu YF, Liu XQ, et al. (2009). Incidence of sudden cardiac death in China: analysis of 4 regional populations. J. Am. Coll. Cardiol. 54: 1110-1118. http://dx.doi.org/10.1016/j.jacc.2009.06.016 
Kostin S, Dammer S, Hein S, Klovekorn WP, et al. (2004). Connexin 43 expression and distribution in compensated and decompensated cardiac hypertrophy in patients with aortic stenosis. Cardiovasc. Res. 62: 426-436. http://dx.doi. org/10.1016/j.cardiores.2003.12.010

Kuniyasu A, Kaneko K, Kawahara K and Nakayama H (2003). Molecular assembly and subcellular distribution of ATP-sensitive potassium channel proteins in rat hearts. FEBS Lett. 552: 259-263. http://dx.doi.org/10.1016/S00145793(03)00936-0

Li J, Tang HQ, Li JH, Zhou ZR, et al. (2011). A meta analysis on effect of Wen Xin Ke Li in treatment of arrhythmia. Chin. J. Evid. Based Cardiovasc. Med. 3: 84-89.

Li LD, Zhang CT, Ruan L, Ni MK, et al. (2010). [Effects of combined amiodarone and antiarrhythmic peptide use on the cardiac gap junctions and incidence of induced ventricular arrhythmias in healed myocardial infarction rabbit models]. Zhonghua. Xin Xue Guan Bing Za Zhi 38: 1102-1107.

Li YF (2009). Cardiogenie sudden death in the elderly. Chin. J. Geriatric Heart Brain Vessel Dis. 11: 932-933.

Lin JJ, Li YG, Tan XR and Zeng X (2003). Impacts of connexin 43 degradation on conduction velocity during acute myocardial ischemia. Chin. J. Pathol. 19: 786-788.

Liu JX, Han X and Ma XB (2005). [Effect of Shuangshen Tongguan Recipe on nuclear factor-kappa B signal pathway and myocardial junction-mediated intercellular communication in acute myocardial ischemia/reperfusion injured model rats]. Zhongguo Zhong Xi Yi Jie He Za Zhi 25: 228-231.

Matsushita K, Yatsuya H and Tamakoshi K (2006). [Epidemiology of acute coronary syndrome]. Nippon Rinsho 64: 625-632.

Peters NS, Coromilas J, Severs NJ and Wit AL (1997). Disturbed connexin43 gap junction distribution correlates with the location of reentrant circuits in the epicardial border zone of healing canine infarcts that cause ventricular tachycardia. Circulation 95: 988-996.http://dx.doi.org/10.1161/01.CIR.95.4.988

Sun B, Qi XQ and Sun BG (2005). Effects of gap junction blocker on ventricular arrhythmias induced by myocardial ischemia. Chin. Circ. J. 8: 260-263.

Tosaki A, Engelman RM and Das DK (1996). Effects of cromakalim and glibenclamide in ischemic and reperfused hearts. Ann. N. Y. Acad. Sci. 793: 460-465. http://dx.doi.org/10.1111/j.1749-6632.1996.tb33542.x

Wang D and Liu Q (2014). Effects of Wenxin Granule on cardiomyocytes in myocardial ischemia-reperfusion injury of rats. Chin. J. Gerontol. 5: 2820-2821.

Wang FM, Wang F and Zhang SH (2013). Connexin remodeling and arrhythmia. Chin. J. Cardiovasc. Rehabil. Med. 22: 626-629.

Wang N, De Vuyst E, Ponsaerts R, Boengler K, et al. (2013). Selective inhibition of Cx43 hemichannels by Gap19 and its impact on myocardial ischemia/reperfusion injury. Basic Res. Cardiol. 108: 309.http://dx.doi.org/10.1007/s00395$\underline{012-0309-\mathrm{X}}$

Wang X, Wang X, Tang YH, Wang T, et al. (2011). Effects of Wenxin granule on L-type calcium current in ventricular myocytes of rat. South China J. Card. Dis. 17: 60-63.

Waza AA, Andrabi K and Hussain MU (2014). Protein kinase C (PKC) mediated interaction between conexin43 (Cx43) and $\mathrm{K}(+)(\mathrm{ATP})$ channel subunit (Kir6.1) in cardiomyocyte mitochondria: Implications in cytoprotection against hypoxia induced cell apoptosis. Cell. Signal. 26: 1909-1917. http://dx.doi.org/10.1016/j.cellsig.2014.05.002

Wu AM, Wang SR, Zhang DM, Zhao MJ, et al. (2007). Effect of Fuzhenghuayu capsule on the expression of connexin 43 in rats after myocardial infarction. Chin. J. Int. Med. Cardio-/Cerebr. Dis 5: 1078-1080.

Xin L, Hou WL and Wang L (2011). An overview on clinical studies of mechanism of Chinese medicine for antiarrhythmic effects. Chin. J. Gerontol. 31: 4287-4290.

Yang J (2013). Influence of Gegen Qinlian decoction on the expression of Cx43 and cardiac enzymes in rat arrhythmia model. Master's thesis, Anhui University of Chinese Medicine (in Chinese).

Yao JH and Gao HCh (2007). Clinical study of the treatment of premature ventricular contraction with Tiao Gan Qing Xin Decoction. Inter. J. Trad. Chin. Med. 29: 67-69.

Yu ZB and Sheng JJ (2011). [Remodeling of cardiac gap junctions and arrhythmias]. Sheng Li Xue Bao 63: 586-592. 